\title{
Editorial
}

\section{Tributo a Mar de Fontcuberta Balaguer (1946-2021)}

Rayén Condeza Dall'Orso, Pontificia Universidad Católica de Chile, Santiago, Chile (rcondeza@uc.cl)

Dedicamos el número 51 de nuestra revista a Mar de Fontcuberta Balaguer, investigadora y profesora titular de la Facultad de Comunicaciones de la Pontificia Universidad Católica de Chile, fallecida el pasado 24 de noviembre en su Barcelona natal. Queremos expresar nuestro profundo pesar por su deceso y rendir tributo, en la medida de lo posible, a nuestra apreciada colega, docente y amiga por su invaluable legado y sus cualidades personales. Doctora en Periodismo y Ciencias de la Comunicación por la Universidad Autónoma de Barcelona, donde fue elegida decana de la Facultad de Ciencias de la Comunicación en 1983, Mar de Fontcuberta Balaguer cofundó el Gabinete de Comunicación y Educación de esa casa de estudios en 1994. En la Pontificia Universidad Católica de Chile fue directora de investigación y posgrado de la Facultad de Comunicaciones, donde creó dos programas de magíster: el de Dirección y edición periodística y el de Comunicación y Educación. Este último, vigente desde hace dos décadas, ha formado a cientos de profesionales de distintas disciplinas y países de la región latinoamericana.

Mar fue también una activa colaboradora de Cuadernos.Info. En este espacio, contribuyó a generar conocimiento en el campo de las comunicaciones desde los distintos ámbitos de su interés. Los archivos de nuestra revista ilustran parte de su valioso trabajo en diversas áreas. Una de sus líneas de investigación fue la de los estudios en periodismo. Consideraba que los periódicos y los medios informativos ocupan un lugar axial en las democracias modernas (Fontcuberta \& Borrat, 2006). Por lo mismo, se adelantó a reflexionar sobre problemáticas como la relación entre la pauta y la calidad informativa (De Fontcuberta, 1999), así como sobre la necesidad de la existencia de los medios de comunicación regionales (De Fontcuberta, 1997). En su artículo sobre los riesgos del periodismo mosaico "que disgrega y desinforma" (De Fontcuberta, 2006, p. 30) propone enseñar el "periodismo sistema, que articula y dota de sentido a los contenidos" (Ibid.). Como editora invitada, lideró el monográfico sobre periodismo intercultural en 2011. En la editorial de ese dossier afirma que este tipo de periodismo es fundamental para abordar el conocimiento de la sociedad actual, compleja y global, "tanto en sus aspectos positivos como en sus distorsiones" (De Fontcuberta, 2011, p. 131). 
Entre los artículos más citados de Cuadernos.Info se encuentra su lúcida reflexión sobre la relación necesaria entre la comunicación y la educación (De Fontcuberta, 2001), tal vez una de sus contribuciones más conocidas a nivel internacional, que pone el acento en la importancia de una educación crítica hacia la lógica de los medios. En esta línea de investigación también publicó sobre la situación de la educación en medios en Chile, estudiada desde la perspectiva de la formación inicial del profesorado en el país de ese entonces (Fontcuberta \& Guerrero, 2007). Su última contribución a esta revista como editora invitada fue dedicada a un dossier sobre la ampliación de las fronteras de la comunicación y de la educación (Condeza \& de Fontcuberta, 2014). Mar también se desempeñó como profesional en distintos medios de comunicación en España, y en Chile se hizo conocida por su participación como comentarista radial de actualidad en Radio Cooperativa.

Los quince artículos que publicamos en este número de Temas Generales habrían interesado a Mar sobremanera por su pertinencia y actualidad. Agrupados en la línea de los estudios sobre el periodismo, la contribución interuniversitaria española de Marcel Mauri Ríos, Xavier Ramón-Vegas, Ruth Rodríguez-Martínez y Jesús Díaz-Campo asocia al periodismo de calidad con la propuesta de un sistema de once indicadores para determinar la capacidad de transparencia, autorregulación y participación de los usuarios en los medios informativos digitales. Para los autores, parte ineludible de la responsabilidad de los medios es involucrarse en la tarea de la rendición de cuentas o accountability.

La problemática de la cobertura desigual por género en México es otra de las líneas de investigación relevantes en los estudios sobre periodismo. La encontramos en el artículo de Elizabeth Tiscareño-García, Santiago Gallur Santorum y Óscar Mario Miranda-Villanueva, contribuyendo desde México y República Dominicana al estudio de la presencia de las fuentes femeninas y masculinas en la cobertura del feminicidio en la prensa mexicana, en un país en el que este tipo de crímenes siguen al alza. A partir del análisis de 2091 fuentes relevadas de noticias publicadas en cuatro periódicos de tirada nacional, se observa una situación paradójica: una discriminación hacia las mujeres como fuente oficial en este tipo de hechos, a pesar de que ellas tuvieron mayor presencia como expertas.

En la misma línea, Ana María Muñoz -Muñoz y Juana Salido-Fernández contribuyen desde España a investigar la representación de las atletas en los Juegos Olímpicos de Río en medios deportivos de alto consumo en España con una discusión de literatura específica en torno al deporte como uno de los espacios culturales de reproducción de estereotipos y roles de género. Los resultados muestran una cobertura desigual de las mujeres, aun cuando las atletas obtienen medallas, así como el refuerzo de que habría ciertos deportes considerados como más adecuados 
para mujeres que para hombres, amplificando de este modo los roles tradicionales femeninos en las distintas manifestaciones deportivas.

Los autores Pedro Moura y Fábio Ribeiro estudian el fenómeno del clickbait (ciberanzuelo) en los titulares de noticias deportivas de los tres sitios más populares y con alta visualización en Portugal como una forma de abordar la problemática de las malas prácticas a las que a veces se recurre para conseguir clics de parte de las audiencias, a pesar de que las informaciones sean falsas. Recurren a un estudio en el que desmenuzan la relación que tienen los títulos estudiados con las noticias que desarrollan, si responden a preguntas relevantes, si informan sobre algún acontecimiento o hecho y si tienden a aludir a emociones, entre otros criterios.

En el mismo ecosistema de medios informativos digitales en cambio y sus desafíos para lograr un periodismo de calidad, María Francisca Greene González, María José Lecaros Menéndez y María Fernanda Cerda Diez investigan las prácticas de elaboración de la pauta periodística en los diez medios digitales con mayor lectoría en Chile durante 2020. A partir de entrevistas a sus editores y directores, se observa que progresivamente ha habido una apertura a incorporar contenidos que pueden considerarse como un reflejo de la influencia de la audiencia. La comparación con estudios previos en el contexto chileno muestra que, en 2020, todos los medios digitales estudiados disponían de una rutina de pauta diaria y que la figura del editor se ha perfilado de manera específica, así como que hay una mayor carga laboral para los periodistas.

En el contexto de la pandemia por COVID-19 se produjo una explosión de estudios en el ámbito de las comunicaciones. Al inicio de la enfermedad expandida a nivel global, la población más representada en los medios fue, de lejos, la de los adultos mayores. El estudio de Virginia Guarinos y de Daniel Martín-Pena analiza la presencia y la representación de las personas mayores de 65 años en la cadena radial y los programas con mayor audiencia en España desde el inicio del confinamiento hasta la primera apertura del país, alterando el punto visto de los estudios etarios. El análisis de la programación estudia los tipos de estereotipos asociados con las personas mayores según las informaciones de actualidad de ese período.

Otro sector altamente estudiado a raíz de la pandemia ha sido el del ejercicio periodístico y las condiciones de trabajo de sus profesionales. Ruth de Frutos, de la Universidad de Málaga, y Sebastián Sanjurjo, de la Federación Internacional de Periodistas de Argentina, reportan los resultados de una encuesta sobre los cambios en las condiciones laborales y la situación emocional en la que participaron 95 periodistas latinoamericanos de 16 países de la región, aplicada entre mayo y junio de 2020. Los profesionales declaran consecuencias psicológicas asociadas 
con la pandemia, así como un aumento importante de la precariedad laboral, entre otras consecuencias.

En septiembre de 2021, la Organización Panamericana de Salud (2021) advirtió que la pandemia por COVID-19 exacerbó los factores de riesgo asociados con el suicidio, precisamente por el empeoramiento de la salud mental de la población. En torno a esta problemática, Rita Araújo y Felisbela Lopes exploran la representación del suicidio en la prensa diaria de Portugal a partir de las recomendaciones internacionales dadas a los periodistas al momento de informar sobre estos hechos. Tras analizar el contenido de noticias específicas sobre suicido, las autoras describen los principales encuadres desde los cuales se informa a las audiencias y su correspondencia con dichas recomendaciones.

En el mismo plano de los estudios sobre cobertura informativa, Vanessa Roger-Monzó investiga el tratamiento de la prensa escrita sobre la producción audiovisual sostenible en España mediante un análisis cuantitativo textual de las noticias aparecidas en medios nacionales y en lengua española sobre las políticas y buenas prácticas aplicadas en filmaciones, así como en festivales de cine. Lo anterior, considerando un contexto de mayor sensibilidad pública y periodística por el impacto en el medio ambiente de la actividad humana, en el marco de los Objetivos de Desarrollo Sostenible de Naciones Unidas.

En el contexto de la emergencia de los influencers en las redes sociales y de su rol como prescriptores de marca en la comunicación estratégica y en la publicidad, Paula Rodríguez y José Sixto-García examinan su impacto en el marketing digital de empresas relacionadas con el turismo en España, considerando que la web ha cambiado la forma de viajar. Su estudio se centra en las diez empresas públicas y privadas con mayor inversión publicitaria en España durante 2019 en YouTube e Instagram. Los autores observan distintos usos según la red social empleada.

En el ámbito de la comunicación política, el marketing digital en las redes sociales es un importante recurso de propaganda en tiempo de elecciones. Frederic GuerreroSolé, Lluís Mas-Manchón y Toni Aira investigan la irrupción del partido populista Vox en Twitter, movimiento de extrema derecha, durante las elecciones de abril y de noviembre de 2019 en España. Con base en un análisis de la estructura de redes y de los patrones de interacción entre los usuarios, e ilustrando su dinámica, los autores observan -entre otros hallazgos- que la irrupción de Vox, al menos en lo referente a las redes de intersección de retuiteadores, altera de manera sensible la estructura en comunidades y la acerca al modelo de cámara de resonancia. El artículo se integra en la línea de estudios recientes sobre el fenómeno del populismo de la extrema derecha en Europa y el rol de las redes (Carral \& Tuñón Navarro, 2020). 
Amador Iranzo y Eva Espinar-Ruiz retoman dos movimientos sociales emblemáticos en España: el 15M, o movimiento ciudadano de los Indignados de mayo 2011, y el movimiento de los Afectados por la Hipoteca (PAH), creado en el año 2009. Analizan la cobertura informativa de ambos, observan una tendencia a su representación positiva y analizan los factores que explicarían dicha óptica, siendo el PAH el movimiento que es representado con mayor grado de empatía en los periódicos estudiados.

La colaboración entre universidades de Colombia y España se centra en el estudio de los efectos de la narración persuasiva en mensajes de comunicación y salud desde la perspectiva de la educación y el entretenimiento. Alicia CameloGuarín, Juan-José Igartua, Jair Vega-Casanova y Jorge Enrique Palacio-Sañudo investigan específicamente el caso de la exposición de la población homosexual masculina a contenidos de promoción de los exámenes de VIH, en la modalidad audiovisual y en la textual, así como la importancia de las narrativas y de los personajes en estos casos.

Dos artículos sobre las series audiovisuales en plataformas de streaming o de televisión en horario de alta audiencia cierran la entrega de este número. Desde México, Karla Contreras Tinoco y Laura Alfaro Beracochea examinan las características identitarias, los roles, los ámbitos de actuación y los estereotipos de género en la serie de ficción televisiva mexicana Club de Cuervos (2015), proyectada en un servicio de streaming. Muestran que los personajes hombres se presentan como consejeros, exitosos, propositivos, abnegados e hipersexualizados, con mayor presencia en el espacio público y más homosocialidad. Las mujeres, en cambio, son representadas como amorosas, tiernas, hipersexualizadas, aparecen más en el espacio privado y tienen más relaciones heterosociales.

Mercedes Herrero De la Fuente, Anto Garzía y María-José Establés investigan el impacto en las redes sociales del spin-off \#Luimelia, una extensión de narrativa transmedia de una de las tramas de la exitosa ficción española Amar es para siempre, la más vista a diario en España, sobre la relación afectivo-amorosa entre dos mujeres. A partir del análisis cuantitativo de su repercusión en Twitter y de tres entrevistas en profundidad semiestructuradas a los dos guionistas de la serie y a una profesional responsable de la medición de audiencias, se revela que la movilización de los fans fue decisiva y cómo su estrategia de redes contribuye a desarrollar la trama y a construir a los personajes protagonistas.

RAYÉN CONDEZA DALL'ORSO, PHD

Editora en jefe Cuadernos.info 


\section{REFERENCIAS}

Carral, U. \& Tuñón-Navarro, J. (2020). Estrategia de comunicación organizacional en redes sociales: análisis electoral de la extrema derecha francesa en Twitter (Organizational communication strategies on social networks: analysis of the French far-right's electoral campaigns on Twitter). El professional de la información, 29(6), e290608. https://doi.org/10.3145/epi.2020.nov.08

Condeza, R. \& de Fontcuberta, M. (2014). Comunicación y Educación: Ampliando las fronteras (Communication and Education: Expanding the Frontiers). Cuadernos.Info, (35), 10-11. https://doi.org/10.7764/cdi.35.22871

De Fontcuberta, M. (1997). La identidad regional de los medios (The Regional Identity of the Media). Cuadernos.Info, (12), 44-50. https://doi.org/10.7764/cdi.12.207

De Fontcuberta, M. (1999). Pauta y calidad informativa (Guideline and informative quality). Cuadernos.Info, (13), 61-69. https://doi.org/10.7764/cdi.13.197

de Fontcuberta, M. (2001). Comunicación y educación: Una relación necesaria (Communication and education: A necessary relationship. Cuadernos.Info, (14). https://doi.org/10.7764/cdi.14.190

De Fontcuberta, M. (2006). Simplificación periodística: la realidad como mosaico. (Journalistic simplification: reality as a mosaic). Cuadernos.Info, (19), 30-35. https://doi.org/10.7764/cdi.19.121

De Fontcuberta, M. (2011). Introducción. Interculturalidad: el gran desafío de los medios. (Introduction. Interculturality: the great challenge of the media). Cuadernos.Info, (29), 131-132. https://doi.org/10.7764/cdi.29.242

De Fontcuberta, M. \& Guerrero, C. (2007). Una nueva propuesta para la educación en medios (A New Proposal for Media Education). Cuadernos.Info , (20), 87-97. https://doi.org/10.7764/cdi.20.115

Fontcuberta, M. \& Borrat, H.(2006). Periódicos: sistemas complejos, narradores en interacción (Newspapers: complex systems, interacting narrators). La Crujía.

Organización Panamericana de la Salud. (2021, September 9). La OPS insta a priorizar la prevención del suicidio tras 18 meses de pandemia por COVID-19 (Suicide prevention must be prioritized after 18 months of COVID-19 pandemic, says PAHO). Paho.org. https://www.paho.org/es/noticias/9-9-2021-ops-insta-priorizar-prevencion-suicidiotras-18-meses-pandemia-por-covid-19 